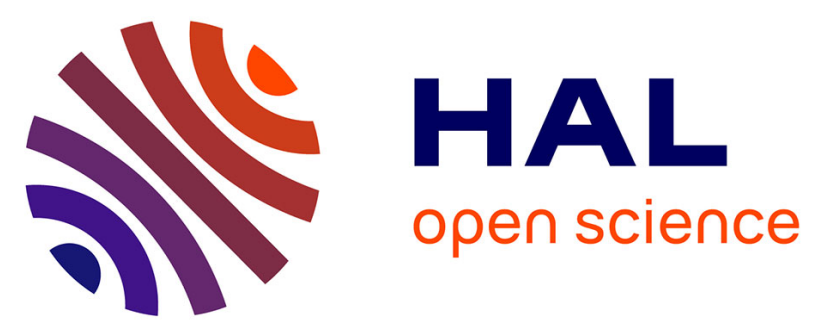

\title{
Neutron Laue and X-ray diffraction study of a new crystallographic superspace phase in n-nonadecane-urea
}

\author{
S. Zerdane, C. Mariette, G. J. Mcintyre, M.-H. Lemée-Cailleau, P. Rabiller,
} Laurent Guérin, J. C. Ameline, Bertrand Toudic

\section{- To cite this version:}

S. Zerdane, C. Mariette, G. J. Mcintyre, M.-H. Lemée-Cailleau, P. Rabiller, et al.. Neutron Laue and X-ray diffraction study of a new crystallographic superspace phase in n-nonadecaneurea. Acta Crystallographica Section B : Structural Science [1968-2013], 2015, 71 (3), pp.293-299. 10.1107/S2052520615005442 . hal-01168334

\section{HAL Id: hal-01168334}

https://hal-univ-rennes1.archives-ouvertes.fr/hal-01168334

Submitted on 26 Jun 2015

HAL is a multi-disciplinary open access archive for the deposit and dissemination of scientific research documents, whether they are published or not. The documents may come from teaching and research institutions in France or abroad, or from public or private research centers.
L'archive ouverte pluridisciplinaire $\mathbf{H A L}$, est destinée au dépôt et à la diffusion de documents scientifiques de niveau recherche, publiés ou non, émanant des établissements d'enseignement et de recherche français ou étrangers, des laboratoires publics ou privés. 


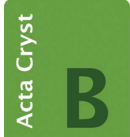

STRUCTURAL SCIENCE CRYSTAL ENGINEERING MATERIALS

ISSN 2052-5206

Received 9 January 2015

Accepted 16 March 2015

Edited by M. Dusek, Academy of Sciences of the Czech Republic, Czech Republic

Keywords: aperiodic composite crystals; symmetry; crystallographic superspace; neutron Laue diffraction.

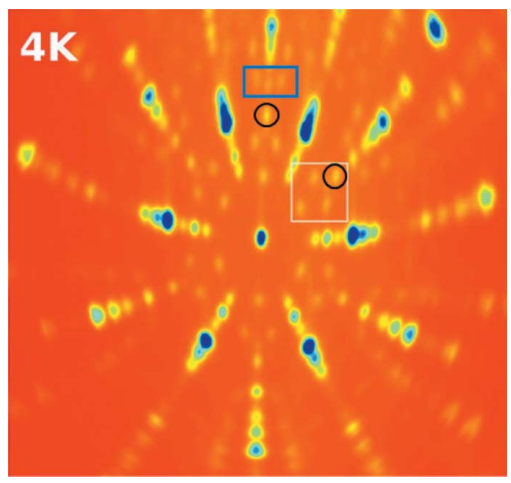

C 2015 International Union of Crystallography

\section{Neutron Laue and X-ray diffraction study of a new crystallographic superspace phase in n-nonadecane-urea}

\author{
S. Zerdane, ${ }^{\text {a }}$ C. Mariette, ${ }^{\text {a }}$ G. J. McIntyre, ${ }^{\text {b }}$ M.-H. Lemée-Cailleau, ${ }^{c}$ P. Rabiller, ${ }^{\text {a }}$ L. \\ Guérin, ${ }^{\mathrm{a}}$ J. C. Ameline ${ }^{\mathrm{a}}$ and B. Toudic ${ }^{\mathrm{a} *}$
} anstitut de Physique de Rennes, UMR UR1-CNRS 6251, Université de Rennes 1, 35042 Rennes, France, ${ }^{\mathbf{b}}$ Australian
Nuclear Science and Technology Organisation, New Illawarra Road, Lucas Heights NSW 223, Australia, and ${ }^{\text {Institut }}$
Laue-Langevin, 71 avenue des Martyrs, 38042 Grenoble CEDEX 9, France. *Correspondence e-mail:
bertrand.toudic@univ-rennes1.fr

Aperiodic composite crystals present long-range order without translational symmetry. These materials may be described as the intersection in three dimensions of a crystal which is periodic in a higher-dimensional space. In such materials, symmetry breaking must be described as structural changes within these crystallographic superspaces. The increase in the number of superspace groups with the increase in the dimension of the superspace allows many more structural solutions. This is illustrated in $n$-nonadecane-urea, revealing a fifth higher-dimensional phase at low temperature.

\section{Introduction}

Confinement of molecules within aperiodic host matrices is of fundamental importance in structural science, supramolecular chemistry and biophysics. In such systems, aperiodicity between the host and guest sublattices appears rather naturally due to the misfit of the different cell parameters along one or more directions. This occurs, for example, when the guests are chains of atoms and ions in organic and inorganic matrices, fullerene molecules in carbon nanotubes, and long confined molecules in host structures. The new definition of the crystal by the International Union of Crystallography is a solid material which has essentially a discrete diffraction pattern, so it includes these materials which present longrange order without translational symmetry. Periodicity in the structure is actually recovered in higher-dimensional spaces, called crystallographic superspaces (Coppens, 1995; Janssen, 2007; Janssen et al., 2006, 2007; van Smaalen, 2007). A crystallographic superspace is characterized by more than three independent basis vectors, the number of these vectors defining the dimension or rank $n$ of the superspace.

For host/guest intergrowth nanotubular structures, which have a sole incommensurate direction $\boldsymbol{c}$, a four-dimensional superspace description usually gives the positions of the complete set of Bragg peaks (Hollingsworth \& Harris, 1996; Janssen et al., 2007; van Smaalen, 2007; van Smaalen \& Harris, 1996)

$$
\mathbf{Q}_{h k l m}=h \mathbf{a}^{*}+k \mathbf{b}^{*}+l \mathbf{c}_{\mathrm{h}}^{*}+m \mathbf{c}_{\mathrm{g}}^{*}
$$

where $\mathbf{a}^{*}, \mathbf{b}^{*}, \mathbf{c}_{\mathrm{h}}^{*}$ and $\mathbf{c}_{\mathrm{g}}^{*}$ are the conventional reciprocal unitcell vectors, and $\mathbf{c}_{\mathrm{h}}$ and $\mathbf{c}_{\mathrm{g}}$ refer, respectively, to the host and the guest parameters along the aperiodic direction. Four indices are needed to describe the four different types of structural Bragg peaks $(h k l m)$ : a convenient but simplistic 


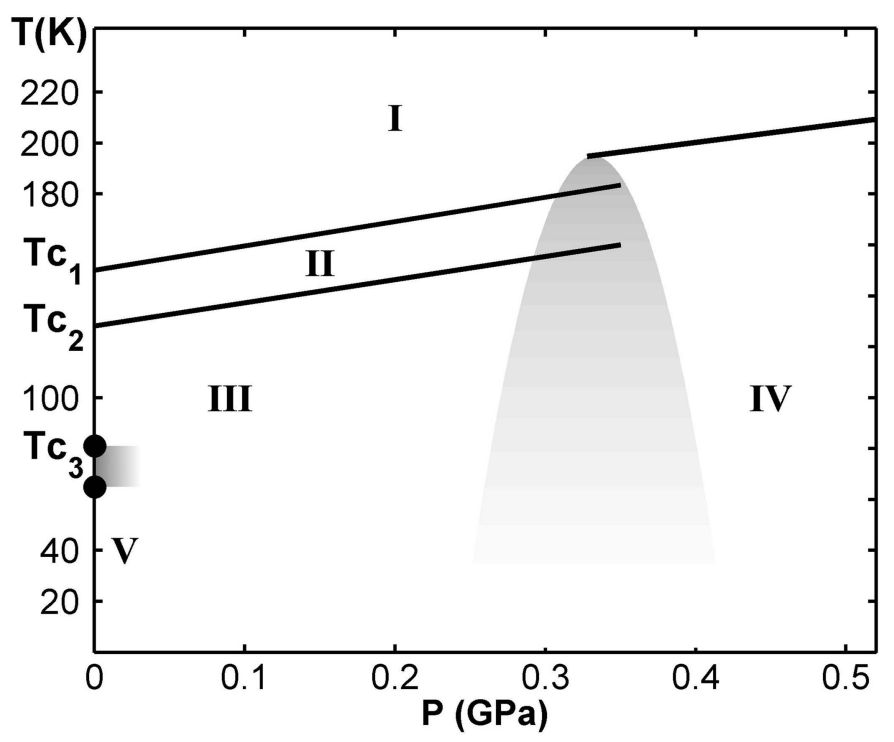

Figure 1

Phase diagram $(T, P)$ of the fully deuterated $n$-nonadecane-urea, as determined by neutron diffraction under pressure (from Toudic et al., 2011). All the phases (I, II, III, IV, V) require a description within a crystallographic superspace. The parabolic shaded region indicates the metastable region between the ordered low-pressure and high-pressure phases. The two points associated with $T_{\mathrm{c} 3}$ mark the metastability limit between phases III and V, as reported in this paper.

labeling is that $(h k 00),(h k l 0),(h k 0 m)$ and $(h k l m)$, with $l$ and $m$ different from zero, are the common, host, guest and satellite Bragg peaks, respectively.

Within this family of aperiodic intergrowth nanotubular structures, the organic guest/host $n$-alkane/urea compounds constitute a prototype family. In almost all of the alkane/urea inclusion compounds, a hexagonal space group is reported in the literature for the host subsystem, with $a_{\text {hex }}=b_{\text {hex }}=8.22 \AA$, and the pitch of the urea helix $c_{\mathrm{h}}=11.02 \AA$ at ambient conditions (Frey \& Boysen, 1998; George \& Harris, 1995; Harris \& Thomas, 1990; Hollingsworth \& Harris, 1996; Lefort et al., 1996; van Smaalen \& Harris, 1996; Weber et al., 1996). In the case of $n$-alkane/urea, an empirical experimental estimation of the guest periodicity $c_{\mathrm{g}}$ was reported by Lenné et al. (1970): $c_{\mathrm{g}}=[(n-1) 1.277+3.48] \AA$, where $n$ is the number of $\mathrm{C}$ atoms in the alkane molecule. Later a mathematical model provided a formalism that allows potential-energy functions (describing host-guest interactions, guest-guest interactions and intramolecular potential energies) computed for any onedimensional inclusion compound to be used to predict the guest periodicity of alkane/urea inclusion compounds (Shannon et al., 1995). The best-fit straight line for optimal $c_{\mathrm{g}}$ versus $n$ is: $c_{\mathrm{g}}=[(n-1) 1.26+3.64] \AA$. In most cases the ratio of the host and guest $c$ parameters is irrational defining a misfit parameter $\gamma=\left(c_{\mathrm{g}}^{*} / c_{\mathrm{h}}^{*}\right)=\left(c_{\mathrm{h}} / c_{\mathrm{g}}\right)$. In this letter we will consider $n$-nonadecane-urea, for which the estimated value of $\gamma$ at room temperature is $0.416-0.419$.

A large amount of work has been dedicated to the phase transitions in this prototype family, but almost all of the first experimental work was described in terms of conventional three-dimensional crystallography, ignoring their aperiodic features. Then a unique phase transition was reported in almost all of these crystals, independently of the $n$-alkane guest. This unique phase transition was considered to be the same one in all compounds, i.e. an improper ferroelastic transition (Forst et al., 1986, 1990; Fukao, 1994a,b; Guillaume et al., 1990; Peral et al., 2001). It was described by a simple spin-phonon model governed by an antiferro-ordering affecting both the host shearing and the guest orientation (Lynden-Bell, 1993). The same phase transition was assumed to occur under pressure and its evolution was established up to $0.2 \mathrm{GPa}$ (Fukao et al., 1990). From a theoretical point of view, a general paper has been dedicated to the phase transitions of such aperiodic crystals (van Smaalen \& Harris, 1996). This analysis is performed in the frame of the superspace crystallographic description and all possible group-sub/group relationships are considered for the host and guest sublattices structures. Such an analysis, done in a four-dimensional crystallographic superspace, does not take into account symmetry breaking driven by the host-guest intermodulation change.

We recently reported a sequence of phases in $n$-nonadecane-urea which involves such structural degrees of freedom (Toudic et al., 2008; Mariette et al., 2013) at atmospheric pressure. The study of the $(T, P)$ phase diagram reported afterwards brings evidence of the increase in the dimensionality of the crystallographic superspace to create new structural solutions within this formalism (Toudic et al., 2011). The phase diagram in Fig. 1 shows the pressure dependence of two different phase transitions, one at $T_{\mathrm{c} 1}$ and another at $T_{\mathrm{c} 2}$, up to $P=0.35 \mathrm{GPa}$. At these pressures the first phase transition, at $T_{\mathrm{c} 1}$, displays a strong ferroelastic feature, characterized by a shearing of the structure in the basal plane (Forst et al., 1990). Above $P=0.35 \mathrm{GPa}$, a different ordered phase appears, called phase IV. This orthorhombic ordered phase does not involve any measurable shearing of the crystal (Bourgeois et al., 2004). The sole remaining transition temperature, $T_{\mathrm{c} 1}$, between 80 and $300 \mathrm{~K}$ continues to increase smoothly with pressure. The phase transformation between the two ordered low-pressure phases and the high-pressure phase reveals a strong metastability, which is illustrated schematically in Fig. 1 by a shaded area (Toudic et al., 2011).

To summarize, the high-symmetry phase I is hexagonal of rank four, $P 6_{1} 22(00 \gamma)$ (Hollingsworth \& Harris, 1996; van Smaalen \& Harris, 1996). The value of the misfit parameter is found to be equal to 0.418 at all temperatures at atmospheric pressure. The superspace group associated with the so-called phase II, between 150 and $130 \mathrm{~K}$ at atmospheric pressure, is of rank five, $C 222_{1}(00 \gamma)(10 \delta)$, where $\delta$ is the magnitude in units of $\mathbf{c}_{\mathrm{h}}^{*}$ of the wavevector of the superspace modulation. The value of $\delta$ was found to be equal to 0.090 at ambient pressure. The phase transition from phase I to phase II presents a clear ferroelastic feature, with the creation of at least six symmetryrelated domains (Fig. 2; Forst et al., 1990). The values of the parameters $\gamma$ and $\delta$ change under hydrostatic pressure (Bourgeois et al., 2003). Under high pressure, at low temperature, a similar superspace group, $C 222_{1}(00 \gamma)(10 \delta)$, is created below $T_{\mathrm{c} 1}$, but without any measurable shearing $\left(\mathbf{a}_{\mathrm{o}}=\mathbf{a}_{\mathrm{h}}, \mathbf{b}_{\mathrm{o}}=\mathbf{a}_{\mathrm{h}}-2 \mathbf{b}_{\mathrm{h}}, b_{\mathrm{o}}=a_{\mathrm{o}}(3)^{1 / 2}\right.$ in the orthorhombic 
description). This phase is historically called phase IV, as reported in Fig. 1 (Toudic et al., 2011). The $(T, P)$ phase diagram shows the pressure dependence of the second phasetransition line at $T_{\mathrm{c} 2}$, at low pressure. This phase transition corresponds to the loss of the C-lattice centering of the fivedimensional superspace group leading to the superspace group $P 2_{1} 2_{1} 2_{1}(00 \gamma)(00 \delta)$, also five-dimensional. It is associated with superstructure Bragg peaks appearing in particular in the $\left(\mathbf{a}^{*}, \mathbf{b}^{*}\right)$ plane (Fig. $\left.2 b\right)$. In this plane the superstructure Bragg peaks satisfy the condition, $h+k$ odd, this being reproduced six times due to the six different symmetryinduced domains (two red, two blue and two green in Fig. 2c; Toudic et al., 2011).

With no evident reason at the time to suspect that further transitions would occur at lower temperature, the studies above were limited to $80 \mathrm{~K}$. In fact, this assumption was unfounded and here we present a study down to $4 \mathrm{~K}$, at atmospheric pressure, in which we describe an additional phase transition within the crystallographic superspaces of five dimensions.

\section{Experimental}

In order to search for new phases below liquid nitrogen temperature, neutron diffraction studies were performed on the Laue diffractometers VIVALDI at the High-Flux Reactor at the Institut Laue-Langevin (ILL), Grenoble, France, and KOALA at the OPAL reactor at the Australian Nuclear Science and Technology Organization (ANSTO), Lucas Heights NSW, Australia. Both diffractometers use thermal neutrons, with wavelengths from 0.8 to $5.2 \AA$, and allow fast survey of reciprocal space over a broad range of temperature down to $2 \mathrm{~K}$. The sample is set at the center of a cylindrical detector composed of neutron-sensitive image plates (McIntyre et al., 2006). With a large solid angle of detection $(\sim 2 \pi$ sterad) a large volume of reciprocal space is observed in a single pattern, which is more than adequate to detect most phase transitions. Single patterns were recorded for one position of the crystal at temperatures from 300 to $2 \mathrm{~K}$, with full data collections of typically 10 patterns distinguished by $20^{\circ}$ rotations of the crystal around the vertical axis at selected temperatures. The analysis of the Laue images has been carried out with the ImageJ package (Rasband, 2014) and the ESMERALDA program suite (Fuentes-Montero et al., 2015).

The determination of the superspace group was then pursued by high-resolution monochromatic X-ray diffraction. $\mathrm{X}$-ray diffraction measurements were performed using monochromated $\mathrm{Cu} K \alpha$ radiation from a rotating-anode source and a high-resolution mar345dtb imaging plate (Marresearch $\mathrm{GmbH}$ ), which was placed as far as $300 \mathrm{~mm}$ from the crystal to resolve the Bragg peaks spatially. Full data acquisitions with 1 or $2^{\circ} \varphi$ rotation steps were used to reconstruct the diffraction planes of interest. CrysAlisPro software (Agilent, 2010) was used to analyze the data.

Neutron studies were made on single crystals of fully deuterated nonadecane-urea prepared by a slow evaporation of a mixed solution of urea and nonadecane in a mixture of ethanol and 2-propanol. X-ray diffraction studies were performed on fully hydrogenated crystals.

\section{Neutron Laue diffraction evidence of a supplementary phase transition below liquid nitrogen temperature}

A key factor in the discovery of the type of transition from phase I to phase II was the examination of the diffraction pattern in three dimensions, using a four-circle triple-axis spectrometer (D10 at the ILL), the detector used in zeroenergy transfer to reduce the background and improve resolution. Despite the greater accessibility possible in monochromatic four-circle geometry, the volume of reciprocal space that could be explored was limited, especially in view of the weak intensity of the superlattice reflections. Modern neutron Laue diffractometers with large image-plate detectors permit

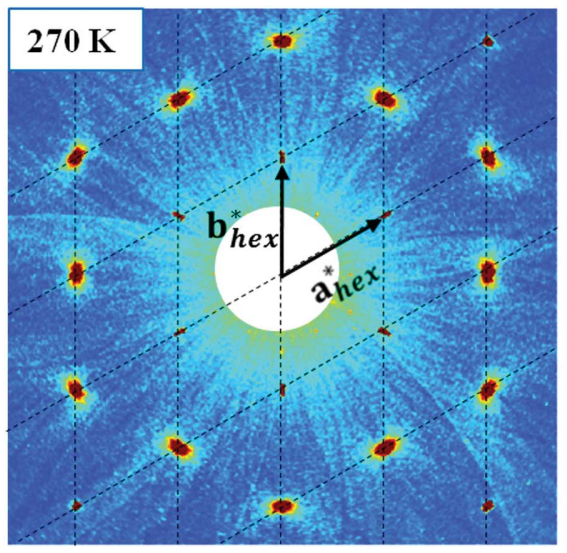

(a)

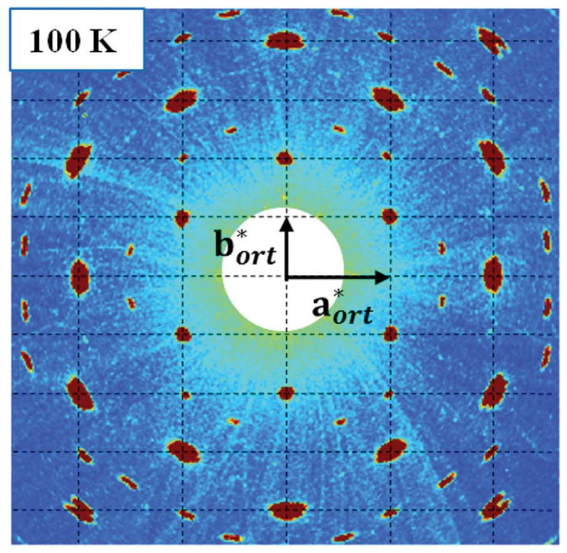

(b)

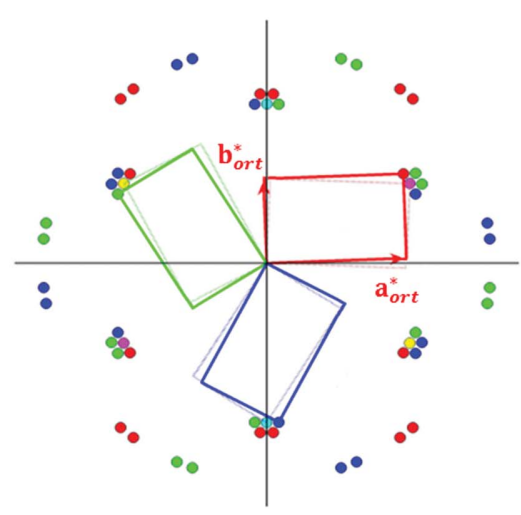

(c)

Figure 2

The reciprocal $\left(\mathbf{a}^{*}, \mathbf{b}^{*}\right)$ plane in $n$-nonadecane-urea: $(a)$ as measured by X-ray diffraction in the hexagonal phase $P 6_{1} 22(00 \gamma)$ at room temperature; $(b)$ as reported in the orthorhombic phase III $P 2_{1} 2_{1} 2_{1}(00 \gamma)(00 \delta) ;(c)$ schematic representation of the $\left(\mathbf{a}^{*}, \mathbf{b}^{*}\right)$ plane, taking into account the systematic extinctions and the six induced ferroelastic domains. 
more extensive and rapid exploration of reciprocal space with good spatial resolution in the two-dimensional projection and a high dynamic range with negligible bleeding of the most intense Laue spots.

Our experiments on VIVALDI at the ILL and KOALA at ANSTO reveal several new unexpected features in the temperature phase diagram of $n$-nonadecane-urea, most notably a new phase (phase V in Fig. 1) below $60 \mathrm{~K}$. Fig. 3 shows the whole set of Bragg peaks present in the $n$-nonadecane/urea Laue diffraction image at one setting of the crystal. In particular, superstructure Bragg peaks which signal the ferroelastic phase transition at $T_{\mathrm{c} 1}$ are shown with black circles. The temperature evolution of the intensity of these characteristic Bragg peaks is presented in Fig. 4(a). They confirm the transition temperature at $T_{\mathrm{c} 1}=150 \mathrm{~K}$. This Laue measurement does not possess a sufficiently good spatial resolution to resolve the Bragg splitting at the ferroelastic hexagonal-to-orthorhombic phase transition. For the same reason, and also because of weak intensities, the second phase transition which corresponds to the loss of the lattice centering within the five-dimensional crystallographic superspace, at $T_{\mathrm{c} 2}$, is also not easily detected (blue rectangles in Fig. 3). The major result is the appearance of a new set of Bragg peaks, which appear in the Laue diffraction image at $4 \mathrm{~K}$ (Fig. 3, within the white square). The temperature dependence of these new Bragg peaks has been obtained by the integration of the intensities within a box of constant area, after background subtraction. Temperature dependences are shown in Fig. 4(b) for decreasing and increasing temperature. In this way, a new ordered phase is reported at lower temperature in $n$-nona-

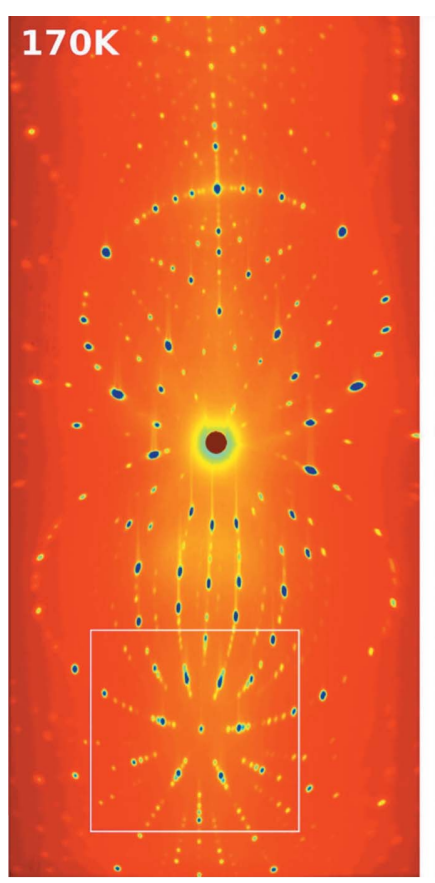

(a)

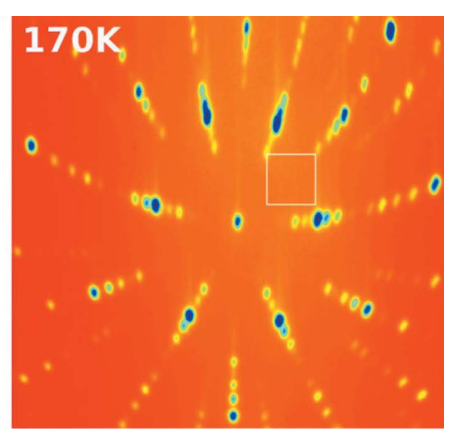

(b)

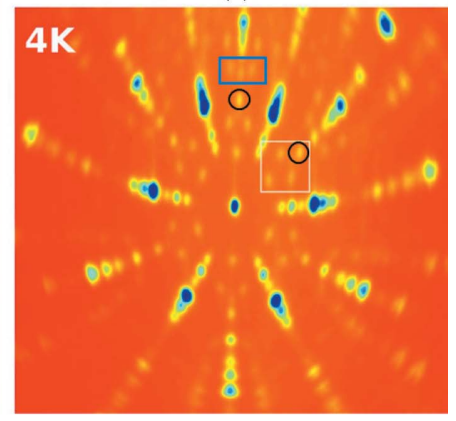

(c)
Figure 3

(a) Laue diffraction image of $n$-nonadecane-urea at $T=170 \mathrm{~K}$ (phase I). (b) Expansion of the selected region of the diffraction image at $170 \mathrm{~K}$; (c) same part of the diffraction image at $4 \mathrm{~K}$. decane-urea. A hysteresis effect is clearly shown, defining $T_{\text {c3down }}=60 \mathrm{~K}$ and $T_{\text {c3up }}=80 \mathrm{~K}$.

Due to chromatic overlap the neutron Laue experiment does not readily allow determination of the indices of the new reflections, and in particular whether they are associated with structural changes in just one of the host or guest sublattices, or with host-guest intermodulation.

\section{High-resolution X-ray characterization of phase V}

In order to analyze this new symmetry breaking, a data collection was performed using high-resolution X-ray diffraction. Such a study allows a separate analysis of the different diffraction planes mentioned above: $\left(\begin{array}{lllll}h & k & 0 & 0 & 0\end{array}\right)$ planes containing Bragg peaks common to urea and alkane networks, $(h k l 00)$ planes containing information on the host reciprocal periodicity, and ( $h k 0 m 0)$ planes containing

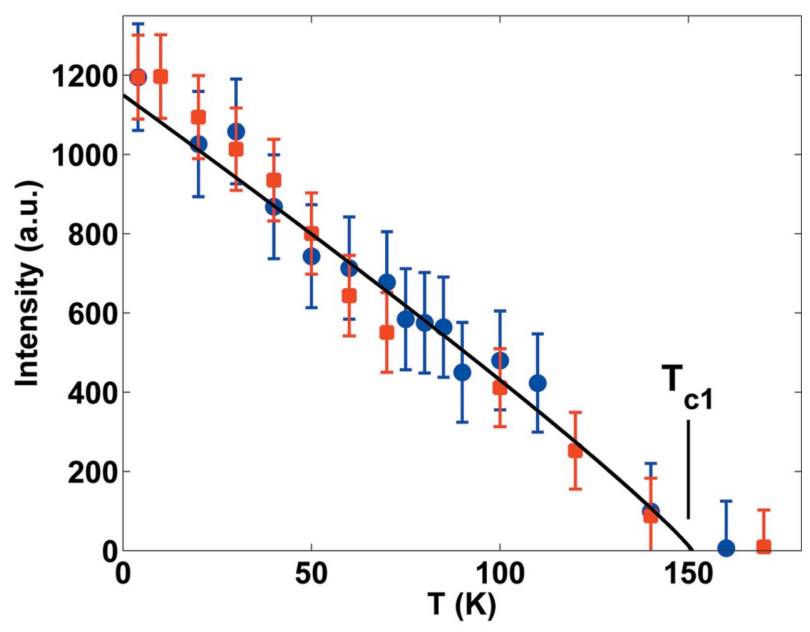

(a)

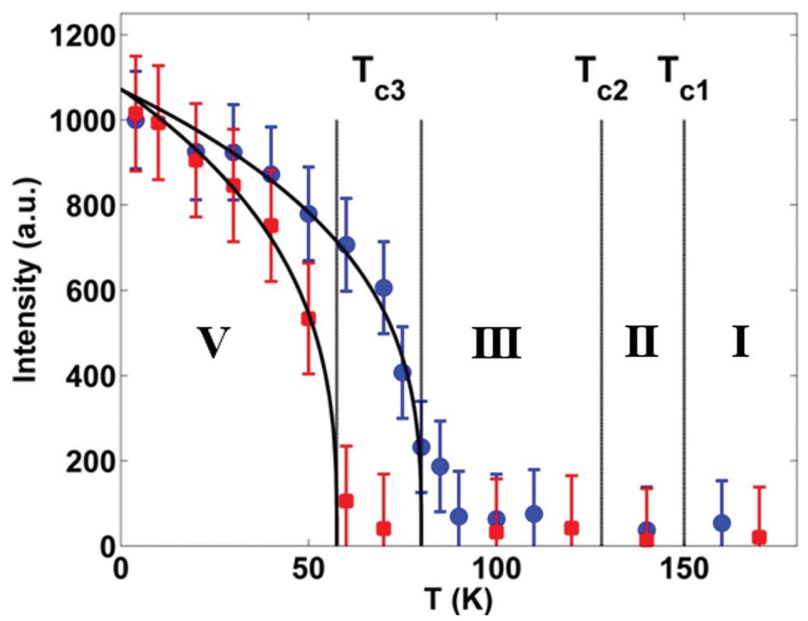

Figure 4

(b)

(a) Temperature evolution of the integrated intensities of Bragg peaks that signal the hexagonal (phase I) to orthorhombic phase (phase II) transition at $T_{\mathrm{c} 1}=150 \mathrm{~K}$ (in blue: heating, in red: cooling). (b) Temperature evolution of the intensities of Bragg peaks that signal the transition from the orthorhombic phase III to a new phase labelled $\mathrm{V}$ at $T_{\mathrm{c} 3}=60 \mathrm{~K}$ upon cooling, with a clear hysteresis effect $\left(T_{\mathrm{c} 3}=80 \mathrm{~K}\right.$ upon heating). 

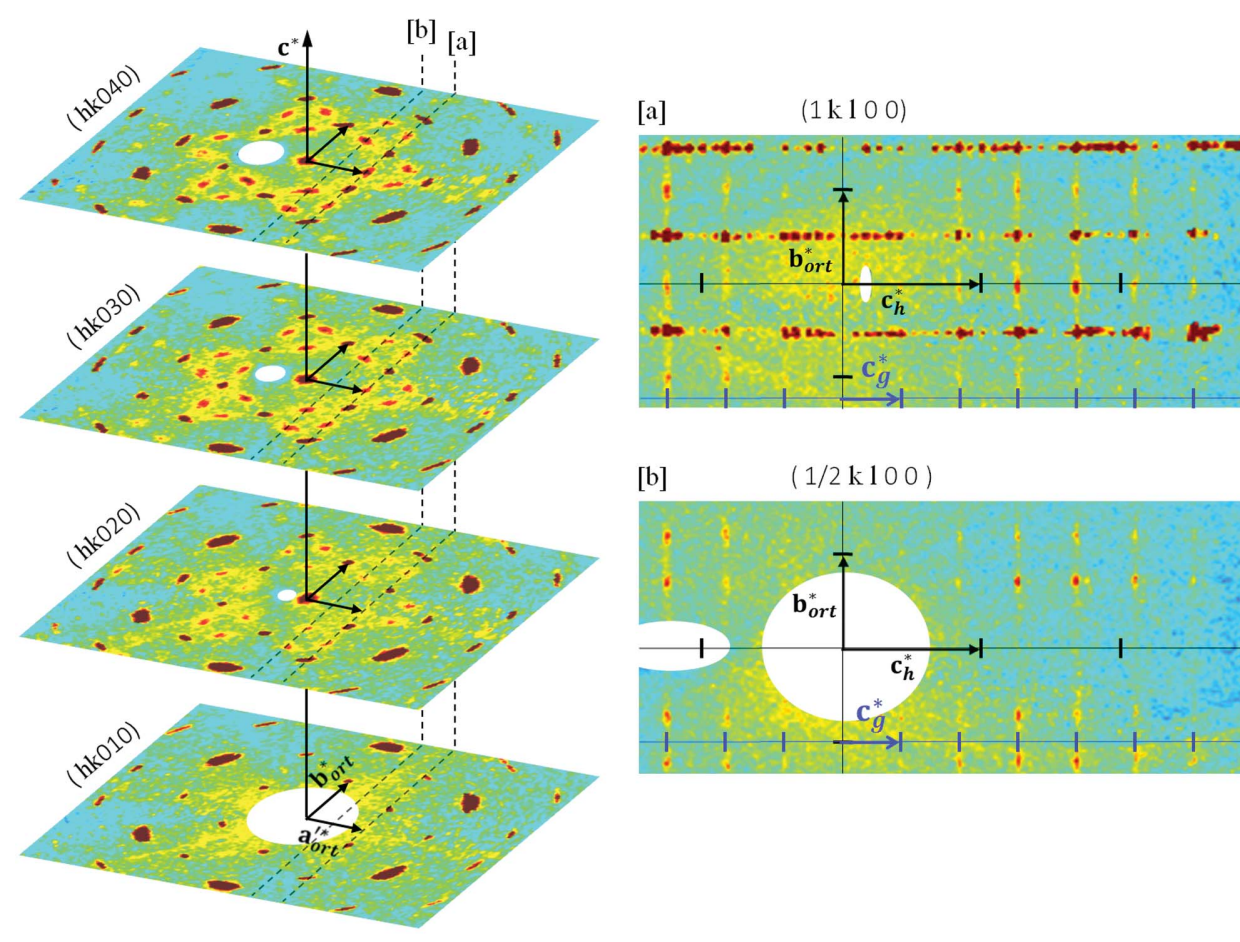

Figure 5

(a)

(b)

(a) Reconstructed reciprocal planes $\left(\mathbf{a}^{*}, \mathbf{b}^{*}\right)$ for $m=1,2,3$ and 4 measured by X-ray diffraction at $10 \mathrm{~K}$ on $n$-nonadecane-urea. (b) Reconstructed reciprocal planes $\left(\mathbf{b}^{*}, \mathbf{c}^{*}\right)$ for $h=1$ and $h=1 / 2$ (dashed lines in the left figure).

information on the guest periodicity, according to the fivedimensional notation.

Reconstructed reciprocal ( $h k 0 m 0)$ planes as measured by $\mathrm{X}$-ray diffraction in $n$-nonadecane-urea at $10 \mathrm{~K}$ are shown in Fig. 5. According to these data for phase V, we have determined the values of the misfit parameters $\gamma$ and $\delta$ defining the periodicity of the complete set of Bragg peaks, as previously reported in phases II and III (Toudic et al., 2008, 2011). The misfit parameter $\gamma$ is found to be equal to $0.418 \pm 0.001$, within the precision limit of our data, a value consistent with those reported previously at ambient pressure for this material. We can then conclude that there is no significant change

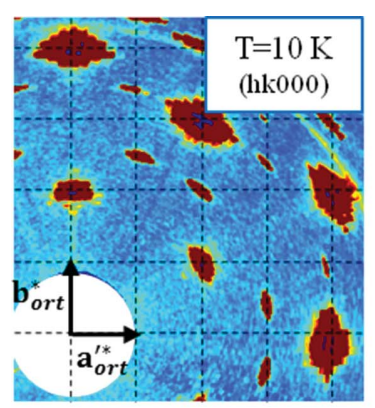

(a)

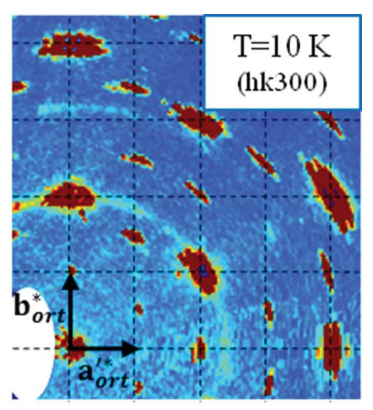

(b)
Figure 6

The reconstructed reciprocal common $(h k 000)$ plane $(a)$ and host $(h k 3$ $00)$ plane $(b)$ as measured by X-ray diffraction in $n$-nonadecane-urea at $10 \mathrm{~K}$. in the ratio of the host and guest misfit parameters at ambient pressure. The fit of the data performed along the superstructure line verifying $h+k$ as odd gives the value of $\delta$ to be $0.090 \pm 0.001$. This value is also consistent with those reported in the two low-temperature phases II and III, again indicating no significant change of $\delta$ with temperature at ambient pressure.

The reconstructed planes at the guest reciprocal periodicity ( $h k 0$ $m 0)$ at $10 \mathrm{~K}$ are shown in the left panel of Fig. 5. The planes ( $h k 02$ $0),(h k 030)$ and ( $h k 040)$ reveal a supplementary dense set of Bragg peaks, not present in phases II and III, as illustrated in Fig. 2(c). This signature in the X-ray diffraction pattern is associated with that reported in the neutron Laue experiment. We note that the intensity of these supplementary Bragg peaks is not observable in the $\left(\mathbf{a}^{*} \mathbf{b}^{*}\right)$ plane, very weak for $m=$ 1 and increases with the value of $m$, being maximum for $m=4$ in the Xray data.

The equivalent reconstructed reciprocal plane, ( $\left.h k \begin{array}{lll}c & 0 & 0\end{array}\right)$ for the common subsystem and ( $\left.h \begin{array}{llll}k & 3 & 0 & 0\end{array}\right)$ for the host reciprocal periodicity, are reported in Fig. 6 at $10 \mathrm{~K}$ in phase V. The analysis reveals no difference for these planes between the two phases III and V. This is experimentally observed for all the different measured ( $h k l 00)$ planes, as seen in Figs. $5(a)$ and $(b)$ on the right, which present new sets of superstructure Bragg peaks only at multiple values of $\mathbf{c}_{\mathrm{g}}^{*}$. It indicates that the host sublattice does not present a structural change in going from phase III to phase V.

The first conclusion of the X-ray study at $10 \mathrm{~K}$ is that the phase transition involves only the alkane guest ordering in the ab plane. The $\left(\mathbf{b}_{\mathrm{o}}^{*}, \mathbf{c}^{*}\right)$ diffraction patterns presented on the right in Fig. 5 definitively show that all the new Bragg peaks characterizing phase $\mathrm{V}$ are located along the $\mathbf{c}^{*}$ direction at multiple values of $\gamma$.

The whole set of data is explained by allowing a doubling along $\mathbf{a}_{\mathrm{o}}$ of the unit cell for the alkane subsystem only. This is represented in Fig. 7(a) where the black points denote the reciprocal points of high-symmetry phase I. The green points indicate the Bragg peaks which appear in phase II of the superspace group $P 2{ }_{1} 2_{1} 2_{1}(00 \gamma)(00 \delta)$. The red spots locate the Bragg peaks which appear in phase $\mathrm{V}$ : they are at positions (h $k 0 m 0$ ) solely. Fig. 7(b) describes the three different symmetry-induced domains resulting from the loss of the hexagonal symmetry and the existence of other twofold symmetry-induced domains as reported by Forst et al. (1990) at $T_{\mathrm{c} 1}$. The values $h=1$ and $h=1 / 2$ in Fig. 5 are given according 


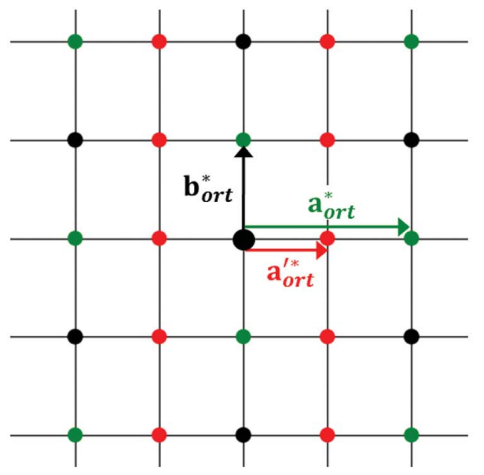

(a)

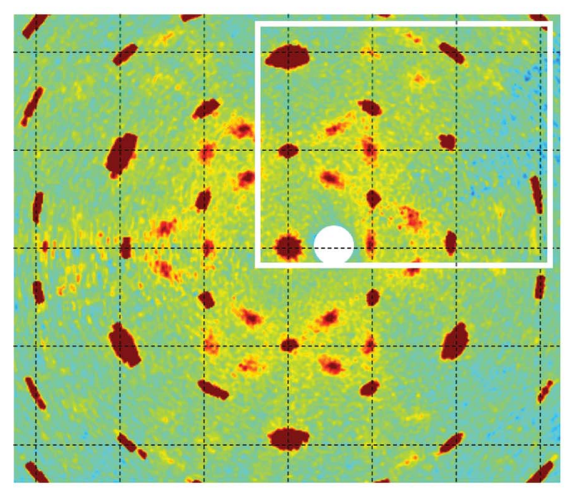

(c)

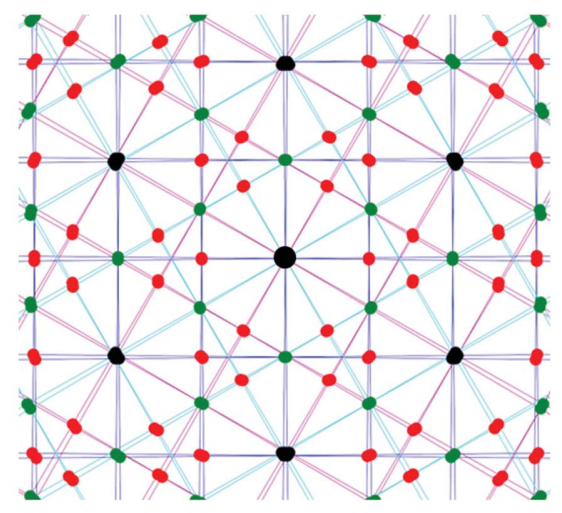

(b)

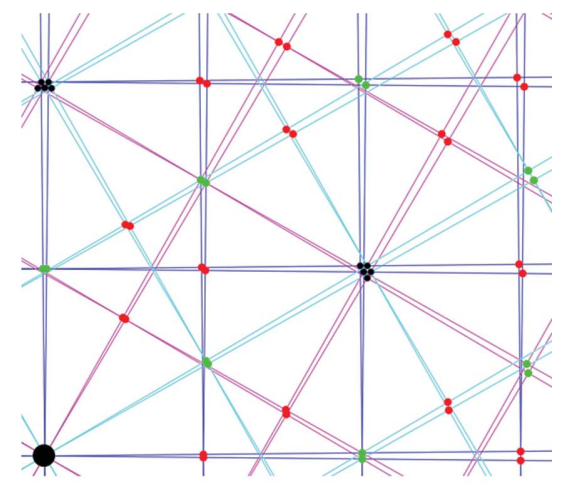

(d)

Figure 7

Schematic representation of ( $a)$ the reciprocal plane $(h k 0 m 0)$ in phase V considering one domain (red points correspond to the new Bragg peaks in this phase, $\left.\mathbf{a}_{\mathrm{o}}^{*^{\prime}}=\mathbf{a}_{\mathrm{o}}^{*} / 2\right) ;(b)$ reciprocal plane ( $\left.k k 0 \mathrm{~m} 0\right)$ in phase $\mathrm{V}$ considering the six possible domains as discussed in Fig. $2(c) ;(c)$ the $(h k 030)$ diffraction plane measured by X-ray diffraction at $10 \mathrm{~K}$ in $n$-nonadecane-urea; $(d)$ predicted diffraction image corresponding to the selected rectangular zone in $(c)$.

to this description of phase V. Fig. 7(c) presents the experimental data measured in a $(h k 0 \mathrm{~m} 0)$ plane, here $(h k 040)$. Full agreement is observed between the predicted (Fig. $7 d$ ) and the observed diffraction patterns (selected rectangular zone in Fig. 7c).

With the knowledge that the additional reflections in phase $\mathrm{V}$ correspond to a doubling of the orthorhombic a axis, and that the magnitude along $\mathbf{c}^{*}$ should be a multiple of 0.418 , the three new neutron reflections shown in the white square in Fig. $3(c)$ can be indexed. One must also take into account the existence of the two additional merohedral domains related by a rotation of 120 or $240^{\circ}$ from the first domain (the supplementary splitting of the Bragg peaks actually yielding six domains is not clearly seen in the Laue experiment due to the limited spatial resolution). The reciprocal units of the three domains are then shown in Fig. 7(b) with three different colors: blue (domain 1), cyan (domain 2) and pink (domain 3). The three Bragg peaks which sign the appearance of phase $\mathrm{V}$ are indexed (1 110210$)$ (from the blue domain 1), (1 - 10210$)$

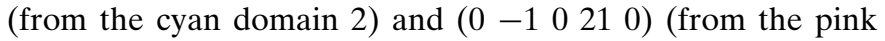
domain 3). The value of $m$ is found to be 21, a value which corresponds to the location in reciprocal space of the maximum of the alkane molecular structure factor. This

maximum originates from the internal periodicity of the alkane molecule which is generated by the linear repetition of the $\mathrm{C}_{2} \mathrm{D}_{4}$ entity (internal periodicity $D=2.56 \AA$ ), limited by its molecular length (Forst et al., 1987). This generates a broad region of diffuse scattering which, for $n$-nonadecane-urea, is centered at the value $m=21$ (Rabiller et al., 2001).

According to the evolution of the intensity of the new Bragg peaks reported by X-ray diffraction as a function of the parameter $m$, we can obtain some information on the structural change at the phase transition. The absence of superstructure Bragg peaks for $l=m=0$ means that there is no component along the $\mathbf{a}$ and $\mathbf{b}$ directions. In other words, we can exclude antiphase displacement of the center of mass or of the mean rotational angle of the guest molecule along the a direction of the orthorhombic plane. On the other hand, the increase of the intensity of the superstructure Bragg peaks up to $m$ $=4$ is in favor of a translational component along the a direction. This displacement is in antiphase from channel to channel along the a axis (Fig. 8). Unfortunately, there are only a few unique new reflections observed by neutrons or $\mathrm{X}$-rays, and the data, even if combined, are presently insufficient to allow refinement of the structure in phase V. Actually, the determination of the structure of the $n$-nonadecane-urea crystals remains a very difficult task in all phases. Most significantly, in the orthorhombic phases, where one has the best chance to solve the structure because of the much smaller dynamic disorder of the guest molecules, the crystal exhibits
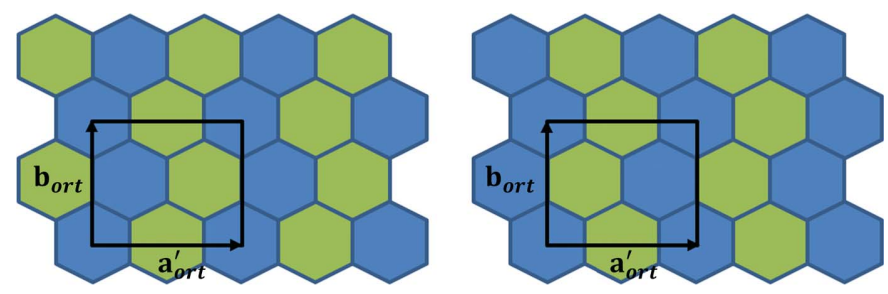

Figure 8

Schematic representation of phase V. The two different colors illustrate the doubling of the unit cell along the a direction of the orthorhombic unit cell $\left(\mathbf{a}_{\mathrm{o}}^{\prime}=2 \mathbf{a}_{\mathrm{o}}\right)$. According to our analysis, the doubling concerns only the guest subsystem with essentially antiphase longitudinal displacement of the alkane along the channel (blue and green, respectively). Two different crystallographically equivalent domains may be generated by this symmetry change (right and left image). 
sixfold non-merohedral twinning. This makes it difficult to scale the data properly for single-crystal structure determination if the domains are not populated equally in different regions of the crystal. With regard to phase I, because of the considerable static and dynamic guest disorder, there are no structural investigations of alkane/urea inclusion compounds in the $P 6_{1} 22(00 \gamma)$ phase that have thus far revealed any detailed structural information about the guest.

\section{Conclusion}

This paper reports the evidence for a new phase in $n$-nonadecane-urea, called phase $\mathrm{V}$, shown initially by Laue neutron diffraction. This transition presents a large hysteresis phenomenon, $T_{\mathrm{c} 3}=60 \mathrm{~K}$ when decreasing temperature and $80 \mathrm{~K}$ when heating. The high-resolution X-ray diffraction study combining a copper source and large-solid-angle detector allows a detailed and precise analysis of the periodicity features of this new phase. As in phases II and III, phase $\mathrm{V}$ is also described within a crystallographic superspace of rank 5. The misfit parameter $\gamma=c_{\text {host }} / c_{\text {guest }}$ is found to be constant at all temperatures and to be equal to $0.418 \pm 0.001$. The fifth parameter, $\delta$, is found to be equal to $0.090 \pm 0.001$, the same value previously reported at ambient pressure in phases II and III. The distinctive feature of phase V is that it involves only a new ordering in the guest sublattice. The phase transition is associated with a doubling of the unit cell along the direction $\mathbf{a}_{\mathrm{o}}$ of one of the orthorhombic domains: $\mathbf{a}_{\mathrm{o}}^{\prime}=2 \mathbf{a}_{\mathrm{o}}$. The maximum superspace group of phase $\mathrm{V}$ is also of five dimensions, $P 2_{1} 2_{1} 2_{1}(00 \gamma)(00 \delta)$, with no significant supplementary deformation of the host subsystem even if the unit cell is doubled compared with phase III. The existence of such a specific degree of freedom of the guest subsystem in this aperiodic inclusion compound should be investigated in other members of the prototype family.

\section{References}

Agilent (2010). CrysAlisPro. Agilent Technologies, Yarnton, England.

Bourgeois, L., Ecolivet, C., Toudic, B., Bourges, P. \& Breczewski, T. (2003). Phys. Rev. Lett. 91, 025504.

Bourgeois, L., Toudic, B., Ecolivet, C., Ameline, J. C., Bourges, P., Guillaume, F. \& Breczewski, T. (2004). Phys. Rev. Lett. 93, 026101. Coppens, P. (1995). Acta Cryst. B51, 402-410.

Forst, R., Boysen, H., Frey, F., Jagodzinski, H. \& Zeyen, C. (1986). J. Phys. Chem. Solids, 47, 1089-1097.

Forst, R., Jagodzinski, H., Boysen, H. \& Frey, F. (1987). Acta Cryst. B43, 187-197.
Forst, R., Jagodzinski, H., Boysen, H. \& Frey, F. (1990). Acta Cryst. B46, 70-78.

Frey, F. \& Boysen, H. (1998). Phase Transitions, 67, 245-276.

Fuentes-Montero, L., Ceřmak, P. \& Rodriguez-Carvajal, J. (2015). Personal communication.

Fukao, K. (1994a). J. Chem. Phys. 101, 7882-7892.

Fukao, K. (1994b). J. Chem. Phys. 101, 7893-7903.

Fukao, K., Horiushi, T., Taki, S. \& Matsushige, K. (1990). Mol. Cryst. Liq. Cryst. B, 180, 405-416.

George, A. R. \& Harris, K. D. M. (1995). J. Mol. Graph. 13, 138141.

Guillaume, F., Sourisseau, C. \& Dianoux, A. (1990). J. Chem. Phys. 93, 3536-3541.

Harris, K. D. M. \& Thomas, J. M. (1990). Faraday Trans. 86, 29852996.

Hollingsworth, M. D. \& Harris, K. D. M. (1996). Comprehensive Supramolecular Chemistry, edited by D. D. MacNicol, F. Toda \& R. Bishop, pp. 177-237. Oxford: Elsevier Science Ltd.

Janssen, T. (2007). Ferroelectrics, 354, 44-51.

Janssen, T., Chapuis, G. \& de Boissieu, M. (2007). Aperiodic Crystals: From Modulated Phases to Quasicrystals. Oxford University Press.

Janssen, T., Janner, A., Looijenga-Vos, A. \& de Wolff, P. M. (2006). International Tables for Crystallography, Vol. C, edited by. A. J. C. Wilson, pp. 907-955. Heidelberg: Springer.

Lefort, R., Etrillard, J., Toudic, B., Guillaume, F., Breczewski, T. \& Bourges, P. (1996). Phys. Rev. Lett. 77, 4027-4030.

Lenné, H. U., Mez, H. C. \& Schlenk Jr, W. (1970). Liebigs Ann. Chem. 732, 70-96.

Lynden-Bell, R. M. (1993). Mol. Phys. 79, 313-321.

Mariette, C., Guerin, L., Rabiller, P., Ecolivet, C., Garcia-Orduna, P., Bourges, P., Bosak, A., de Sanctis, O., Hollingsworth, M. D., Janssen, T. \& Toudic, B. (2013). Phys. Rev. B, 87, 104101.

McIntyre, G. J., Lemée-Cailleau, M.-H. \& Wilkinson, C. (2006). Physica B, 385-386, 1055-1058.

Peral, I., Madariaga, G., Petricek, V. \& Breczewski, B. (2001). Acta Cryst. B57, 378-385.

Rabiller, P., Etrillard, J., Toupet, L., Kiat, J. M., Launois, P., Petricek, V. \& Breczewski, T. (2001). J. Phys. Condens. Matter, 13, 16531668.

Rasband, W. S. (2014). ImageJ. US National Institutes of Health, Bethesda, Maryland, USA, http://imagej.nih.gov/ij/.

Shannon, I. J., Harris, K. D. M., Guillaume, F., Bocanegra, E. H. \& MacLean, E. J. (1995). J. Chem. Soc. Chem. Commun. pp. 23412342.

Toudic, B., Garcia, P., Odin, C., Rabiller, P., Ecolivet, C., Collet, E., Bourges, P., McIntyre, G. J., Hollingsworth, M. D. \& Breczewski, T. (2008). Science, 319, 69-71.

Toudic, B., Rabiller, P., Bourgeois, L., Huard, M., Ecolivet, C., McIntyre, G. J., Bourges, P., Breczewski, T. \& Janssen, T. (2011). Europhys. Lett. 93, 16003.

van Smaalen, S. (2007). Incommensurate Crystallography. Oxford University Press.

van Smaalen, S. \& Harris, K. D. M. (1996). Proc. R. Soc. London A, 452, 677-700.

Weber, T., Boysen, H., Frey, F. \& Neder, R. (1996). Z. Kristallogr. 211, 238-247. 